\title{
Impact of Pharmacist Previsit Input to Providers on Chronic Opioid Prescribing Safety
}

\author{
Nicholas Cox, PharmD, Casey R. Tak, BA, MPH, Susan E. Cochella, MD, MPH, \\ Eric Leishman, PharmD, and Karen Gunning, PharmD, BCPS, BCACP, FCCP
}

Introduction: Primary care providers (PCPs) account for half of opioid prescriptions, often feel chronic pain patients are challenging to manage, and there is wide variability in practice patterns. The purpose of this pilot study was to evaluate the impact of a previsit pharmacist review of high-risk patients treated with opioids for chronic pain on compliance to guideline recommendations at a family medicine residency clinic.

Methods: All adult patients with an appointment for chronic pain who were prescribed $>50$ morphine milligram equivalents (MMEs)/day had charts reviewed by a pharmacist before each appointment; recommendations were sent electronically to the provider before the appointment. After 4 months of implementation, each patient's chart was manually reviewed to gather outcome variables. The primary outcomes were the mean MMEs/day and pain scores.

Results: Pharmacist previsit recommendations were provided for 45 patients. When comparing outcomes before and after intervention, the mean MMEs/day decreased by $14 \%(P<.001)$, with no change in pain scores $(P=.783)$. Statistically significant improvements were noted in multiple other secondary opioid safety outcomes.

Conclusion: Clinical pharmacists providing previsit recommendations was associated with decreased opioid utilization with no corresponding increase in pain scores and increased compliance to guideline recommendations. (J Am Board Fam Med 2018;31:105-112.)

Keywords: Benzodiazepines, Chronic Pain, Drug Monitoring, Naloxone, Opioid Analgesics, Pain Management, Pharmacists, Pilot Study, Prescriptions, Primary Health Care, Quality Improvement, Referral and Consultation

Over the past decade, the rate of opioid prescribing has increased substantially. In 2012, an estimated 259 million opioid prescriptions were written. ${ }^{1}$ Deaths due to opioid overdose have quadrupled since 1999 , and more than 28,000 deaths occurred

This article was externally peer reviewed.

Submitted 24 May 2017; revised 28 August 2017; accepted 1 September 2017.

From the Department of Pharmacy Services (NC, KG) and Department of Family and Preventive Medicine (SEC), University of Utah Health, Salt Lake City; the Department of Pharmacotherapy, University of Utah College of Pharmacy, Salt Lake City (NC, CRT, EL, KG); and the Department of Family and Preventive Medicine, University of Utah School of Medicine, Salt Lake City (SEC, KG).

Funding: NC, CRT, and EL received financial support for this work through a research grant from the ASHP Research and Education Foundation.

Conflict of interest: none declared.

Corresponding author: Nicholas Cox, PharmD, Department of Pharmacy Services, University of Utah Health, 50 N. Medical Drive, A-090M, Salt Lake City, UT 84132 (E-mail: nicholas.cox@pharm.utah.edu). in 2014. ${ }^{2,3}$ The oft-named "opioid epidemic" has prompted increased health care discussion, federal legislation, and guidelines, published by the Centers for Disease Control and Prevention (CDC), for prescribing opioids for chronic pain. ${ }^{4,5}$

The 2016 CDC guidelines recommend multiple strategies to improve therapy and safety when prescribing opioids for chronic pain. ${ }^{5}$ Recommendations include combined use of nonopioid pharmacologic therapy for patients who are prescribed opioids, reassessment of benefits and risks in patients with doses $>50$ morphine milligram equivalents (MME)/day, avoidance of doses >90 MME/ day, offering outpatient naloxone to patients at high risk of opioid overdose, use of prescription drug monitoring programs at least every 3 months, use of urine drug testing at least annually, avoidance of concurrent use of opioids and benzodiazepines, and use of evidence-based treatment for patients with opioid use disorder (eg, methadone, 
buprenorphine, naltrexone, psychosocial treatment), among others.

Primary care providers (PCPs) write approximately half of all opioid prescriptions in the United States. ${ }^{6}$ PCPs are often the first contact for patients with chronic pain; however, PCPs often feel that these patients are challenging to manage, and practice patterns, documentation, and compliance with opioid prescribing guidelines vary widely. ${ }^{6-10}$ Many PCPs are unaware of or are underusing tools and resources such as opioid prescribing guidelines, pain assessment scales, opioid abuse risk assessment scales, and urine drug screens. ${ }^{8,11-14}$ The 2016 CDC guidelines "refer to and promote integrated pain management and collaborative working relationships with other providers (eg, behavioral health providers, pharmacists, and pain management specialists)." However, the impact and best strategies for pharmacist integration in chronic pain management is largely unknown and unstudied, particularly in primary care settings.

Pharmacist involvement in team-based care for patients with other disease states (eg, diabetes, hypertension, anticoagulation) has been shown to improve patient outcomes, reduce cost, improve adherence, increase patient understanding, and reduce physician burden. ${ }^{15-21}$ Because of the need for monitoring and clinician follow-up, the unique pharmacologic properties, and the potential for diversion, opioid therapy in chronic pain presents an opportunity for pharmacist involvement. The current literature on pharmacist engagement in chronic pain management is limited to specialty care settings (eg, palliative care), investigations of patient-centered medical home designation, or descriptive reports. ${ }^{15,16,22-25}$ To our knowledge, this is the first published study of the impact of a pharmacist-led intervention focused on comprehensive chronic pain management in a family medicine setting. The purpose of this study was to evaluate the impact of a previsit pharmacist review of highrisk patients treated with opioids for chronic noncancer pain for compliance with CDC recommendations at a family medicine residency clinic.

\section{Methods}

\section{Setting and Patients}

This study was conducted at a family medicine residency clinic staffed by 17 attending physicians, 12 family medicine residents, 1 sports medicine fellow, 3 physician assistants, 2 dieticians, 1 psychologist, 1 social worker, 1 care manager, and 2 clinical pharmacists. The clinic conducts approximately 150 patient visits each day and prescribes opioids for chronic pain for approximately $250 \mathrm{pa}$ tients. This study included patients $\geq 18$ years of age who had an appointment for chronic pain during the study period (November 2016 to February 2017) and had been prescribed >50 MME/day (based on prescription directions or the number of pills prescribed per month) as of the most recent clinic appointment before the intervention. Both prescription directions and number of pills prescribed per month were used because prescribers often titrate and taper by altering the number of pills prescribed without altering the prescription directions, especially for medications with "as needed" directions. The MME per day based on prescription directions for medications with "as needed" directions was calculated using the maximum number of pills per day that the directions allow. A 4-month intervention period was selected because this is a pilot study, it was designed as a rapid-cycle quality improvement project, and extensive pharmacist resources were required. $\mathrm{Pa}$ tients prescribed opioids to treat pain associated with cancer or palliative care were excluded. Patients were identified by manually calculating the MME per day for all patients who had completed an opioid treatment agreement. Completion of an opioid treatment agreement is a clinic requirement for all patients who are prescribed opioids for chronic use. The electronic health record (EHR) was manually reviewed for patients prescribed $>50$ MME/day to identify all other inclusion and exclusion criteria. Conversion values used to calculate MME are listed in Appendix 1. All study activities were reviewed and approved by the health system's institutional review board and deemed exempt.

\section{Intervention and Outcomes}

The clinic pharmacists reviewed the schedule of patients meeting inclusion criteria each week to identify patients who had upcoming provider appointments for chronic pain. Appointments for chronic pain were identified by reviewing the prior provider notes for each patient and the clinic scheduling documentation. Approximately 1 to 4 days before the appointment, 1 of the clinic pharmacists reviewed the patient's EHR, summarized 
pain conditions and other relevant health conditions, outlined the patient's current pharmacotherapy plan, and provided recommendations to optimize pain management and opioid prescribing safety. The pharmacists then documented this information in the EHR using a standardized note template and sent it electronically to the provider; when possible, they also discussed it verbally with the provider the day of the appointment. Although the pharmacists had contact with some patients for nonintervention purposes (eg, medication history, education, management of other disease states), the pharmacists did not implement any of the intervention recommendations; implementation was deferred to the prescriber. After 4 months of following of this process, each patient's EHR was manually reviewed by an investigator (EL) who did not participate in the intervention in order to retrospectively collect patient characteristics and study outcomes; a separate investigator (NC) reviewed a sample of patients to ensure accuracy.

The primary outcomes were the mean MME per day (based on prescription directions and the number of pills prescribed per month) and pain scores (on a 1-to-10 scale). Adherence to opioid prescriptions was verified at each appointment. Secondary outcomes included the mean number of nonopioid analgesics prescribed to each patient and the proportion of patients who were concurrently prescribed opioids with benzodiazepines, hypnotics (eg, zolpidem, eszopiclone), or muscle relaxants; were offered an outpatient naloxone prescription; had completed a urine drug screen within the prior 12 months; had a completed and documented review of the state's prescription drug monitoring program within the prior 3 months; had a current or past referral to a pain specialist or physical therapy; and were prescribed a bowel regimen. All secondary outcomes evaluated in this study are based on recommendations or considerations outlined in the CDC guidelines. ${ }^{5}$

For each patient, study outcomes were collected on the day of their first intervention appointment, before implementation of the previsit pharmacist recommendations (referred to as "before intervention"). Study outcomes were collected again in February 2017 after 4 months of pharmacists providing previsit recommendations (referred to as "after intervention"). Outcomes for pharmacist and provider time and effort were also collected.

\section{Data Analysis}

Outcomes were analyzed by comparing each patient's before and after intervention data. Patient characteristics were summarized using descriptive statistics. The Wilcoxon signed rank test was used to compare continuous outcomes and the McNemar test was used to compare categorical outcomes. Although all patients had a pain score documented before the intervention, not all patients had a second appointment with a provider during the study period and thus an after-intervention pain score was not available; as a result, only patients with data available before and after the intervention were included in the analysis of this outcome. This sample size provided power $(80 \%)$ to detect an absolute mean difference in pain scores of 1.1 ; the investigators deemed an absolute mean difference $>1$ to be clinically meaningful. Data were analyzed using SAS version 9.4 (SAS Institute, Cary, NC).

\section{Results}

During the 4-month implementation of this quality improvement initiative, pharmacist previsit recommendations were provided for 45 patients. Patient characteristics are summarized in Table 1 . The majority of patients $(84 \%)$ had multiple documented pain diagnoses; the most common pain diagnosis was unspecified joint pain (67\%). The majority of patients had documented tobacco use (62\%), anxiety (60\%), and depression (56\%). Overall, 38 patients $(84 \%)$ had a documented psychiatric diagnosis.

Results for primary outcomes are summarized in Table 2. When comparing before intervention to after intervention, the mean MME per day based on prescription directions decreased by $17 \%(P<$ .001 ), and the mean MME per day based on the number of pills prescribed per month decreased by $14 \%(P<.001)$. The analysis of MME per day excluded 1 patient prescribed methadone, whose MME per day was reduced by approximately 720 mg. This patient was excluded to avoid an overstated reduction in this outcome. Of the 27 patients with documented pain scores after intervention, no statistically significant change was found in mean pain scores $(P=.783)$.

Results for secondary outcomes are summarized in Table 3. Based on after-intervention data, the most common opioids prescribed were oxycodone (49\% of patients), hydrocodone (27\%), extended- 
Table 1. Patient Demographics

\begin{tabular}{lc}
\hline Characteristics & Patients (n $=45)$ \\
\hline Age (years), mean (SD) & $57(13)$ \\
Female sex & $22(49)$ \\
Race/ethnicity & \\
White & $40(89)$ \\
Hispanic & $3(7)$ \\
Other & $4(9)$ \\
Native American & $1(2)$ \\
Pain indication* & \\
Unspecified joint pain & $30(67)$ \\
Neuropathy & $17(38)$ \\
Unspecified back pain & $15(33)$ \\
Unspecified muscle pain & $11(24)$ \\
Unspecified pain & $11(24)$ \\
Osteoarthritis & $10(22)$ \\
Spinal disease or pain & $9(20)$ \\
Unspecified neck pain & $7(16)$ \\
Fibromyalgia & $3(7)$ \\
Migraine & $2(4)$ \\
Rheumatoid arthritis & $2(4)$ \\
Other & $8(18)$ \\
Comorbidities* & \\
Tobacco use & $28(62)$ \\
Anxiety & $27(60)$ \\
Depression & $25(56)$ \\
Insomnia & $20(44)$ \\
Alcohol use & $18(42)$ \\
Migraines & $9(20)$ \\
History of illicit drug use & $5(11)$ \\
Bipolar & $3(7)$ \\
Other psychiatric condition & $2(4)$ \\
\hline &
\end{tabular}

Data are $\mathrm{n}(\%)$ unless otherwise indicated.

${ }^{*}$ Characteristics are not mutually exclusive. Patients may have multiple documented indications and comorbidities. $\mathrm{SD}$, standard deviation.

release morphine (20\%), and tramadol (16\%). The most common nonopioid analgesics prescribed were acetaminophen $(64 \%)$, oral nonsteroidal antiinflammatory drugs (42\%), gabapentin/pregabalin
(38\%), tricyclic antidepressants (31\%), and topical nonsteroidal anti-inflammatory drugs (27\%).

Pharmacist and provider effort and time are summarized in Table 4 . Of the 118 provider appointments that occurred during the study period, $87(74 \%)$ were with physicians, $20(17 \%)$ were with advanced practice clinicians, and $11(9 \%)$ were with medical residents. Of the 301 recommendations provided, 114 (38\%) were implemented. No difference was found in the proportion of recommendations implemented when stratified by provider type $(P=.925)$. The most common recommendations provided were to initiate/change nonopioid analgesic therapy (100\% of patients), consider an opioid taper $(96 \%)$, refer the patient to a pain specialist (93\%), and offer an outpatient naloxone prescription $(82 \%)$. Of the recommendations provided, those most commonly implemented were to offer an outpatient naloxone prescription (54\%), complete a urine drug screen (52\%), taper opioid therapy $(51 \%)$, and initiate/change nonopioid analgesic therapy (49\%). Appendix 2 summarizes the types of select recommendations, the frequency they were suggested, and the frequency they were implemented.

\section{Discussion}

In this retrospective analysis of a pilot project, a pharmacist providing previsit recommendations to optimize pain management and opioid prescribing safety was associated with decreased opioid use, with no corresponding increase in pain scores. Based on CDC recommendations to avoid $>90$ MME/day, the study population could be considered a "high-risk" population for opioid toxicity as the baseline mean value was $135 \mathrm{MME} /$ day. ${ }^{5}$ Pharmacist recommendations to consider reducing the opioid dose also included a patient-specific taper schedule and nonopioid analgesic options. The in-

Table 2. Morphine Milligram Equivalents per Day and Pain Scores

\begin{tabular}{lcrr}
\hline Outcomes & Before Intervention $(\mathrm{n}=45)$ & After Intervention $(\mathrm{n}=45)$ & $P$ Value \\
\hline MMEs/day & & & \\
Based on prescription directions & $151(110)$ & $125(114)$ & $<.001$ \\
Based on number of pills prescribed per month & $135(100)$ & $116(106)$ & $<.001$ \\
Pain scores* & $5.3(2.6)$ & $5.5(2.5)$ & .783 \\
\hline
\end{tabular}

Data are mean (standard deviation) unless otherwise indicated.

*Only patients with data available before and after the intervention were included in analysis of this outcome $(\mathrm{n}=27)$.

MME, morphine milligram equivalent. 
Table 3. Secondary Outcomes

\begin{tabular}{lrrr}
\hline Outcomes & Before Intervention $(\mathrm{n}=45)$ & After Intervention $(\mathrm{n}=45)$ & $P$ Value \\
\hline Patients concurrently taking high-risk medications & & & $14(31)$ \\
$\quad$ Benzodiazepines & $21(47)$ & $7(16)$ & .008 \\
$\quad$ Hypnotics & $10(22)$ & $10(22)$ & .083 \\
$\quad$ Muscle relaxants & $8(18)$ & $2.4(1.4)$ & .157 \\
Nonopioid analgesics prescribed, mean (SD) & $2.1(1.3)$ & $1.5(0.7)$ & .002 \\
Opioid analgesics prescribed, mean (SD) & $1.6(0.6)$ & $22(49)$ & .219 \\
Offered outpatient naloxone prescription & $6(13)$ & $27(60)$ & .009 \\
Current urine drug screen* & $15(33)$ & $26(58)$ & $<.001$ \\
Current review of the state's PDMP & & $21(47)$ & .046 \\
Referral to pain specialist & $12(27)$ & $34(76)$ & .317 \\
Referral to physical therapy & $17(38)$ & $22(49)$ & $<.001$ \\
Patients prescribed a bowel regimen & $33(73)$ & $6(13)$ & \\
\hline
\end{tabular}

Data are $\mathrm{n}(\%)$ unless otherwise indicated.

* "Current" is defined as completed within the preceding 12 months.

t"Current" is defined as completed and documented within the preceding 3 months.

PDMP, prescription drug monitoring program; SD, standard deviation.

crease in nonopioid analgesics prescribed may have helped offset opioid dose reductions, resulting in a nonsignificant change in pain scores. Because of the small number of patients with documented pain scores after the intervention, this outcome may not have been adequately powered to detect a difference of less than 1.1.

With regard to concurrent use of opioids with other high-risk medications, benzodiazepine use was the only outcome to have a statistically significant change. Of the 3 classes of medications evaluated in this study, benzodiazepines are the only class the CDC guidelines specifically recommend to avoid concurrently prescribing with opioids. ${ }^{5}$

Table 4. Pharmacist and Provider Effort and Time

\begin{tabular}{lc}
\hline Outcomes & Value \\
\hline Pharmacist & \\
$\quad$ Total reviews provided, $\mathrm{n}$ & 80 \\
$\quad$ Reviews per patient & $1.8(0.9)$ \\
$\quad$ Minutes required for review & $33(19)$ \\
Pharmacist recommendations & \\
$\quad$ Total recommendations provided, n & 301 \\
$\quad$ Total recommendations implemented, & $114(38)$ \\
$\quad$ n (\% of total recommendations provided) & \\
$\quad$ Provided per patient & $6.3(1.5)$ \\
$\quad$ Implemented per patient & $2.5(1.3)$ \\
Provider & \\
$\quad$ Total appointments, $\mathrm{n}$ & 118 \\
Appointments per patient & $2.6(1.3)$ \\
\hline
\end{tabular}

Data are mean (standard deviation) unless otherwise indicated.
Nearly half of patients were concurrently prescribed benzodiazepines at baseline, which is likely explained by the large number of patients with documented anxiety. Pharmacist recommendations to discontinue benzodiazepines or hypnotics also included a patientspecific taper schedule (if applicable) and alternative options to treat anxiety and/or insomnia (eg, selective serotonin reuptake inhibitors, hydroxyzine, buspirone, tricyclic antidepressants).

With the exception of concurrent use of hypnotics and muscle relaxants, the mean number of opioids prescribed, and referrals to physical therapy, all other secondary outcomes showed statistically significant improvements. The lack of improvement in concurrent hypnotic and muscle relaxant use may have been affected by the small proportion of patients receiving concurrent therapies at baseline. In a similar way, the lack of improvement in referrals to physical therapy may be secondary to the large proportion of patients who had already been referred. It should be noted that although only $38 \%$ of total recommendations were implemented by providers, the provider acceptance rate was likely higher; many recommendations require substantial time to implement (eg, benzodiazepine taper), and often multiple provider visits are required to implement all planned changes. In addition to providing an evaluation of pharmacist engagement in chronic pain management in a primary care setting, this study also provides a descriptive report of base- 
line pain management practices with regard to guideline recommendations.

Certain clinic characteristics may have contributed to the success of the pharmacist intervention. As a family medicine residency clinic, the clinic has a culture of learning and frequently engages in clinic quality improvement projects. The clinic has a strong interdisciplinary health care focus and has had a clinical pharmacist for over 20 years. In addition, given the recent national attention on opioid prescribing, providers may have been more aware of the need for practice change. Based on the pharmacist time required for each review, this intervention is feasible to implement at other clinics. Given the results of this study, the pilot site's health system is currently working to implement this intervention in all its community clinics. We hypothesize that this pharmacist engagement model can be applicable to a wide variety of primary care settings. Future studies should seek to validate the results of this study on a larger scale.

The results of this study are consistent with other investigations of pharmacist-led interventions in the palliative care and oncology care settings. ${ }^{16,22,23}$ Wilson et $\mathrm{al}^{23}$ reported that implementation of pharmacist recommendations in a palliative care setting was 1 of the strongest predictors of patients achieving desired outcomes. Because the purpose of this study was to evaluate the impact of a previsit pharmacist review, and in an attempt to evaluate an intervention that could feasibly be added to other current pharmacist clinic responsibilities, the pharmacists did not implement any of the intervention recommendations but rather deferred implementation to the prescriber. Other settings may consider adapting the intervention, as several recommendations could have implemented by the pharmacists, for example, through the use of standing orders. For clinics without a pharmacist, computer alert systems and other team members could be used to identify patients and potential interventions. However, we hypothesize that the success of this intervention is a result of the pharmacists providing patient-specific solutions. For example, while another team member could identify patients concurrently taking opioids and benzodiazepines and note that a urine drug screen was due, most nonphysicians do not have the training of pharmacists that would allow them to provide a benzodiazepine taper schedule and potential alternatives for anxiety that are appro- priate for the particular patient, and to interpret the obtained urine drug screen. However, although other team members and computer alert systems may not be able to completely replicate pharmacist involvement, they could be used to streamline the process to decrease the pharmacist's time per patient.

Our study has several limitations. First, the method used to identify the patient population (ie, manual calculation of MME per day for patients who had signed an opioid treatment contract) may not be feasible to use at clinics looking to implement this pharmacist intervention. Other clinics may not require opioid treatment contracts nor have the personnel and time resources to manually calculate MME per day for patients. Second, given the recent national attention on opioid prescribing, the results may have been partially affected by prescribers who were self-motivated to change opioid prescribing practices. During the study period, however, no other quality improvement projects focused on pain management or opioids were occurring at the clinic. Third, opioid use was based on prescription directions and the number of pills prescribed per month, combined with patient confirmation of adherence to the regimen. Prescription directions represent the maximum potential MME on a given day, and the number of pills prescribed per month represent the average MME per day. However, these, along with patients' report of adherence, are not perfect measures of use and could result in information bias. Finally, because some patients called to schedule same-day appointments, a small number of patients did not have pharmacist previsit recommendations provided before their appointment and, as a result, were excluded from this analysis. In addition, patients were identified from among those who had completed an opioid treatment agreement; although a clinic requirement, it is possible that a small number of patients had not completed the agreement and were inappropriately excluded. Based on internal clinic reports, however, $>95 \%$ of patients chronically prescribed opioids had completed the agreement.

\section{Conclusions}

In a family medicine residency clinic, provision of previsit recommendations by clinical pharmacists for patients at high risk of opioid toxicity was associated with decreased opioid use with no corresponding increase in pain scores, decreased concur- 
rent opioid and benzodiazepine use, increased nonopioid analgesic use, and increased compliance with CDC guideline recommendations. This intervention could be used at other primary care clinics to optimize chronic pain management and opioid prescribing safety.

To see this article online, please go to: http://jabfm.org/content/ 31/1/105.full.

\section{References}

1. Paulozzi LJ, Mack KA, Hockenberry JM. Vital signs: variation among states in prescribing of opioid pain relievers and benzodiazepines-United States, 2012. MMWR Morb Mortal Wkly Rep 2014;63:563-8.

2. Centers for Disease Control and Prevention. Understanding the epidemic. Available from: http://www. cdc.gov/drugoverdose/epidemic/index.html. Last updated and reviewed August 20, 2017. Accessed July 29, 2016.

3. Centers for Disease Control and Prevention. Opioid overdose. Available from: http://www.cdc.gov/ drugoverdose/index.html. Last updated and reviewed October 23, 2017. Accessed July 29, 2016.

4. Library of Congress. S.524 - Comprehensive Addiction and Recovery Act of 2016, Pub. L. No. 114198, 114th Congress. Available from: https://www. congress.gov/bill/114th-congress/senate-bill/524/ text. Published July 22, 2016. Accessed July 22, 2016.

5. Dowell D, Haegerich TM, Chou R. CDC guideline for prescribing opioids for chronic pain - United States, 2016. MMWR Recomm Rep 2016;65:1-49.

6. Levy B, Paulozzi L, Mack KA, Jones CM. Trends in opioid analgesic-prescribing rates by specialty, U.S., 2007-2012. Am J Prev Med 2015;49:409-13.

7. Breuer B, Cruciani R, Portenoy RK. Pain management by primary care physicians, pain physicians, chiropractors, and acupuncturists: a national survey. South Med J 2010;103:738-47.

8. Leverence RR, Williams RL, Potter M, et al. Chronic non-cancer pain: a siren for primary care-a report from the Primary Care Multiethnic Network (PRIME Net). J Am Board Fam Med 2011;24:551-61.

9. Anderson D, Wang S, Zlateva I. Comprehensive assessment of chronic pain management in primary care: a first phase of a quality improvement initiative at a multisite community health center. Qual Prim Care 2012;20:421-33.

10. Green CR, Wheeler JR, Marchant B, LaPorte F, Guerrero E. Analysis of the physician variable in pain management. Pain Med 2001;2:317-27.

11. Wolfert MZ, Gilson AM, Dahl JL, Cleary JF. Opioid analgesics for pain control: Wisconsin physicians' knowledge, beliefs, attitudes, and prescribing practices. Pain Med 2010;11:425-34.
12. Clark JD. Chronic pain prevalence and analgesic prescribing in a general medical population. J Pain Symptom Manage 2002;23:131-7.

13. Elder NC, Simmons T, Regan S, Gerrety E. Care for patients with chronic nonmalignant pain with and without chronic opioid prescriptions: a report from the Cincinnati Area Research Group (CARinG) Network. J Am Board Fam Med 2012;25:652-60.

14. Starrels JL, Becker WC, Weiner MG, Li X, Heo M, Turner BJ. Low use of opioid risk reduction strategies in primary care even for high risk patients with chronic pain. J Gen Intern Med 2011;26:958-64.

15. DiPaula BA, Menachery E. Physician-pharmacist collaborative care model for buprenorphine-maintained opioid-dependent patients. J Am Pharm Assoc 2015;55:187-92.

16. Ma JD, Tran V, Chan C, Mitchell WM, Atayee RS. Retrospective analysis of pharmacist interventions in an ambulatory palliative care practice. J Oncol Pharm Pract 2016;22:757-76.

17. Richardson TE, O'Reilly CL, Chen TF. A comprehensive review of the impact of clinical pharmacy services on patient outcomes in mental health. Int J Clin Pharm 2014;36:222-32.

18. Bultman DC, Svarstad BL. Effects of pharmacist monitoring on patient satisfaction with antidepressant medication therapy. J Am Pharm Assoc 2002;42:36-43.

19. Bungay KM, Adler DA, Rogers WH, et al. Description of a clinical pharmacist intervention administered to primary care patients with depression. Gen Hosp Psychiatry 2004;26:210-8.

20. Doherty RB, Crowley RA. Principles supporting dynamic clinical care teams: an American College of Physicians position paper. Ann Intern Med 2013; 159:620-6.

21. Giberson S, Yoder S, Lee MP. Improving patient and health system outcomes through advanced pharmacy practice. A report to the U.S. Surgeon General. Office of the Chief Pharmacist. U.S. Public Health Service, December 2011. Available from https://dcp.psc.gov/OSG/pharmacy/sc_comms_ sg_report.aspx. Accessed November 13, 2017.

22. Valgus J, Jarr S, Schwartz R, Rice M, Bernard SA. Pharmacist-led, interdisciplinary model for delivery of supportive care in the ambulatory cancer clinic setting. J Oncol Pract 2010;6:e1-4.

23. Wilson S, Wahler R, Brown J, Doloresco F, Monte SV. Impact of pharmacist intervention on clinical outcomes in the palliative care setting. Am J Hosp Palliat Care 2011;28:316-20.

24. Thompson CA. Pharmacist, physician collaborate at clinic to treat opioid dependence. Am J Health Syst Pharm 2016;73:738-9.

25. Elder N, Penm M, Pallerla H, et al. Provision of Recommended Chronic Pain Assessment and Management in Primary Care: Does Patient-Centered Medical Home (PCMH) Recognition Make a difference? J Am Board Fam Med 2016;29:474-81. 
Appendix 1. Morphine Milligram Equivalents

Conversion

\begin{tabular}{lc}
\hline Opioid & $\begin{array}{c}\text { Equivalent } \\
\text { Milligram (mg) }\end{array}$ \\
\hline Codeine, oral & 200 \\
Fentanyl, transdermal & 12.5 \\
Hydrocodone, oral & 30 \\
Hydromorphone, oral & 7.5 \\
Hydromorphone, parenteral & 1.5 \\
Methadone, oral (1 to $20 \mathrm{mg} /$ day) & 7.5 \\
Methadone, oral (21 to $40 \mathrm{mg} / \mathrm{day})$ & 3.75 \\
Methadone, oral (41 to $60 \mathrm{mg} / \mathrm{day})$ & 3 \\
Methadone, oral $(61+\mathrm{mg} / \mathrm{day})$ & 2.5 \\
Morphine, oral & 30 \\
Morphine, parenteral & 10 \\
Oxycodone, oral & 20 \\
Oxymorphone, oral & 10 \\
Oxymorphone, parenteral & 1 \\
Tramadol, oral & 120 \\
\hline
\end{tabular}

Appendix 2. Select Recommendations Given and Implemented

\begin{tabular}{lcr}
\hline Select Recommendations & $\begin{array}{c}\text { Given to Provider, } \\
\text { n (\% of patients) }\end{array}$ & $\begin{array}{c}\text { Implemented, } \mathrm{n} \text { (\% of specific } \\
\text { recommendation) }\end{array}$ \\
\hline Initiate/change non-opioid analgesic regimen & $45(100)$ & $22(49)$ \\
Consider opioid taper & $43(96)$ & $22(51)$ \\
Refer to pain specialist & $42(93)$ & $5(12)$ \\
Offer outpatient naloxone prescription & $37(82)$ & $20(54)$ \\
Review state's PDMP & $35(78)$ & $15(43)$ \\
Obtain urine drug screen & $31(69)$ & $16(52)$ \\
Taper/discontinue concurrent high-risk medications & $20(44)$ & $8(40)$
\end{tabular}

PDMP, prescription drug monitoring program. 